\title{
Large Time Behavior of the Vlasov-Poisson-Boltzmann System
}

\author{
Li Li, ${ }^{1}$ Shuilin Jin, ${ }^{2}$ and Li Yang ${ }^{3}$ \\ ${ }^{1}$ Natural Science Research Center, Harbin Institute of Technology, Harbin 150080, China \\ ${ }^{2}$ Department of Mathematics, Harbin Institute of Technology, Harbin 150080, China \\ ${ }^{3}$ Department of Foundation, Harbin Finance University, Harbin 150030, China
}

Correspondence should be addressed to Li Li; lilihit@126.com

Received 18 May 2013; Accepted 1 July 2013

Academic Editor: Daniel C. Biles

Copyright (C) $2013 \mathrm{Li} \mathrm{Li}$ et al. This is an open access article distributed under the Creative Commons Attribution License, which permits unrestricted use, distribution, and reproduction in any medium, provided the original work is properly cited.

The motion of dilute charged particles can be modeled by Vlasov-Poisson-Boltzmann system. We study the large time stability of the VPB system. To be precise, we prove that when time goes to infinity, the solution of VPB system tends to global Maxwellian state in a rate $O\left(t^{-\infty}\right)$, by using a method developed for Boltzmann equation without force in the work of Desvillettes and Villani (2005). The improvement of the present paper is the removal of condition on parameter $\lambda$ as in the work of $\operatorname{Li}(2008)$.

\section{Introduction}

Large time behavior for the Boltzmann equation and related systems is an important topic for both physicists and mathematicians. We consider the Cauchy problem for VlasovPoisson-Boltzmann system in a torus $\mathbb{T}^{N}$ :

$$
\begin{gathered}
f_{t}+v \cdot \nabla_{x} f+\nabla_{x} \phi \cdot \nabla_{v} f=Q(f, f), \quad \text { on } \mathbb{T}^{N}, \\
\Delta \phi=\int_{\mathbb{R}^{N}} f d v-\rho_{0}, \quad \text { on } \mathbb{T}^{N}, \\
f(0, x, v)=f_{0}(x, v), \\
Q(f, f)=\int_{\mathbb{R}^{N}} \int_{\mathbb{S}^{N-1}}\left(f^{\prime} f_{*}^{\prime}-f f_{*}\right) q\left(v-v_{*}, \sigma\right) d \sigma d v_{*} .
\end{gathered}
$$

$f=(t, x, v)$, which represents the distribution of particles, is a function of time $t \in \mathbb{R}^{+}$, particle velocity $v \in \mathbb{R}^{N}$, and position $x \in \mathbb{T}^{N}$. The force $\nabla \phi$ in (1) is controlled by Poisson equation (2), which comes intrinsically by the nonequilibrium distribution of particles.

The quadratic term $Q(f, f)$ is the collision operator and $q\left(v-v_{*}, \sigma\right)$ is the corresponding cross-section. It is wellknown by the conservation of mass that $\rho_{0}=\int_{\mathbb{T}^{N} \times \mathbb{R}^{N}} f_{0} d x d v$ is a fixed constant which represents the background charge.
Without loss of generality, we can assume $\left|\mathbb{T}^{N}\right|=1, \rho_{0}=$ 1. Define $\rho, u, T$, which are functions of $t$ and $x$ by

$$
\begin{gathered}
\rho=\int_{\mathbb{R}^{N}} f d v, \quad \rho u=\int_{\mathbb{R}^{N}} f v d v, \\
\rho|u|^{2}+N \rho T=\int_{\mathbb{R}^{N}} f|v|^{2} d v .
\end{gathered}
$$

Physically, they represent the macroscopic quantities: density, bulk velocity, and temperature, respectively. It is well known that the conservation of mass, momentum, and energy holds:

$$
\begin{gathered}
\frac{d}{d t} \int_{\mathbb{T}^{N}} \rho d x=0, \\
\frac{d}{d t} \int_{\mathbb{T}^{N}} \rho u d x=0, \\
\frac{d}{d t} \int_{\mathbb{T}^{N}}\left(\frac{\rho|u|^{2}}{2}+\frac{N \rho T}{2}+\frac{|\nabla \phi|^{2}}{2}\right) d x=0 .
\end{gathered}
$$

Here, the total energy consists of the kinetic energy $\rho|u|^{2} / 2$, the internal heat energy $N \rho T / 2$, and the electric potential 
energy $|\nabla \phi|^{2} / 2$. By simple translation and dilation, $\rho, u, T$ can be normalized as

$$
\begin{gathered}
\int_{\mathbb{T}^{N} \times \mathbb{R}^{N}} f d v d x=\int_{\mathbb{T}^{N}} \rho d x=\rho_{0}=1, \\
\int_{\mathbb{T}^{N} \times \mathbb{R}^{N}} f v d v d x=\int_{\mathbb{T}^{N}} \rho u d x=0, \\
\int_{\mathbb{T}^{N} \times \mathbb{R}^{N}}\left(f \frac{|v|^{2}}{2}+\frac{|\nabla \phi|^{2}}{2}\right) d v d x \\
=\int_{\mathbb{T}^{N}}\left(\frac{\rho|u|^{2}}{2}+\frac{N \rho T}{2}+\frac{|\nabla \phi|^{2}}{2}\right) d x=\frac{N}{2} .
\end{gathered}
$$

If the initial datum $f_{0}$ satisfies the conservation laws (7), then the stationary solution is a global Maxwellian $M$, in the form of

$$
M=M_{[1,0,1]}=\frac{1}{(2 \pi)^{N / 2}} \exp \left\{-\frac{|v|^{2}}{2}\right\}
$$

where the subscript $[1,0,1]$ represents the corresponding macroscopic quantities: density, bulk velocity, and temperature, respectively.

Traditional method for studying the asymptotic behavior is using linearization around local or global Maxwellian state. Without external force, Ukai [1] proved an exponential decay rate for the cutoff hard potential in a torus in 1974. In 1980, Caflisch [2] obtained a rate like $O\left(e^{-t^{\beta}}\right)$ for the cutoff soft potential with $\gamma \geq-1$ in a torus, where $\beta=2 /(2-\gamma) \in[0,1]$. Strain and Guo [3] extend Caflisch's result in 2008 and get a convergence rate like $O\left(e^{-t^{P}}\right)(0<P<1)$ for the very soft potential case $(\gamma<-1)$. The previous results all make use of the linearization.

However, by using some estimates on systems of secondorder differential inequalities, Desvillettes and Villani [4] obtain an almost exponential convergence rate like $O\left(t^{-\infty}\right)$. The result is weaker than using linearization, but the smallness assumption on initial data $f_{0}-M$ is removed and the conclusion holds for noncutoff collision kernels as well.

Our work is inspired by the work of Desvillettes and Villani [4]. We extend their result for Boltzmann equation without external force to the Vlasov-Poisson-Boltzmann system.

In a previous work [5], the Vlasov-Poisson-Boltzmann system with (2) replaced by

$$
\lambda \Delta \phi=\int_{\mathbb{R}^{N}} f d v-\rho_{0}
$$

is proved to satisfy the following theorem.

Theorem 1. Let $q\left(v-v_{*}, \sigma\right)$ satisfy

$$
q \geq K_{B} \min \left(\left|v-v_{*}\right|^{\gamma_{-}},\left|v-v_{*}\right|^{-\beta_{-}}\right),
$$

and let the collision operator satisfy

$$
\|Q(g, h)\|_{L^{2}\left(\mathbb{R}_{v}^{N}\right)} \leq C_{B}\|g\|_{H_{s_{0}}^{k_{0}}\left(\mathbb{R}_{v}^{N}\right)}\|h\|_{H_{s_{0}}^{k_{0}}\left(\mathbb{R}_{v}^{N}\right)},
$$

for some $k_{0}, s_{0} \geq 0$, where $K_{B}$ and $C_{B}$ are positive constants. Let $(f)_{t \geq 0}$ be a smooth solution of the problem (1), (9), and (3), such that, for all $k, s>0$,

$$
\sup _{t \geq 0}\|f\|_{H_{s}^{k}\left(\mathbb{T}^{N} \times \mathbb{R}^{N}\right)} \leq C_{k, s}<+\infty
$$

and for all $t>0, x \in \mathbb{T}^{N}$, and $v \in \mathbb{R}^{N}$,

$$
f(x, v) \geq K_{0} e^{-A_{0}|v|^{q_{0}}} \quad\left(A_{0}, K_{0}>0 ; q_{0} \geq 2\right) .
$$

Then $\exists \lambda_{0}$, such that, for all $\lambda>\lambda_{0}$, the solution $f$ converges to $M$ in an almost exponential rate; that is, for any small positive constant $\epsilon>0$,

$$
\|f-M\|=O(1) t^{-1 / 700 \epsilon}
$$

where $O(1)$ depends on $K_{B}, \gamma_{-}, \beta_{-}, C_{B}, k_{0}, s_{0}, C_{k, s}, K_{0}, A_{0}, q_{0}$, and $\epsilon$.

The present paper extends the result of [5] by removing the condition on $\lambda$ and considers system (1)-(3). To be precise, the main result of this paper is as follows.

Theorem 2. Under condition (10)-(13), the solution $f$ of problem (1)-(3) converges to $M$ in an almost exponential rate; that is, for any small positive constant $\epsilon>0$,

$$
\|f-M\|=O(1) t^{-1 / 700 \epsilon}
$$

where $O(1)$ depends on the constants in (10)-(13) and $\epsilon$.

Now, we state some results on the existence of solutions of VPB system. The global existence of solutions is proved in [6] in a torus and [7-9] in the whole space with small perturbed initial data. The existence result in [7] also holds for a more general case, like the Vlasov-Maxwell-Boltzmann system.

The following is devoted to the proof of Theorem 2. Section 2 gives some lemmas which will be used later. Proof of the main result is given in Section 3.

\section{Preliminaries}

First, denote some local Maxwellian states in forms of $\rho, u, T$. Define $M_{[\rho, u, T]}, M_{[\rho, u,\langle T\rangle]}, M_{[\rho, 0,\langle T\rangle]}, M_{[\rho, 0,1]}$ as follows:

$$
\begin{aligned}
M_{[\rho, u, T]}(v) & =\frac{\rho}{(2 \pi T)^{N / 2}} \exp \left\{-\frac{|v-u|^{2}}{2 T}\right\}, \\
M_{[\rho, u,\langle T\rangle]}(v) & =\frac{\rho}{(2 \pi\langle T\rangle)^{N / 2}} \exp \left\{-\frac{|v-u|^{2}}{2\langle T\rangle}\right\}, \\
M_{[\rho, 0,\langle T\rangle]}(v) & =\frac{\rho}{(2 \pi\langle T\rangle)^{N / 2}} \exp \left\{-\frac{|v|^{2}}{2\langle T\rangle}\right\}, \\
M_{[\rho, 0,1]}(v) & =\frac{\rho}{(2 \pi)^{N / 2}} \exp \left\{-\frac{|v|^{2}}{2}\right\},
\end{aligned}
$$

where $\langle T\rangle=\int \rho T d x$ stands for the mean temperature.

As we will show in Section 3, the gradient of temperature prevents $f$ from being close to $M_{[\rho, u, T]}$ for too long; 
the symmetric gradient of velocity prevents $f$ from being close to $M_{[\rho, u,\langle T\rangle]}$ for long, that is, the local Maxwellians with constant temperature; and finally, the gradient of $\rho$ and $\phi$ prevents $f$ from being close to $M_{[\rho, 0,\langle T\rangle]}$ and $M_{[\rho, 0,1]}$ for long. In order to estimate the distance between two distributions, we need to define $H$ functional and relative information (or relative entropy) between two distributions, which is the main measure of the distance between $f$ and the local Maxwellians.

Definition 3. Suppose $f$ and $g$ are two distributions on $\mathbb{T}^{N} \times$ $\mathbb{R}^{N}$, s.t.:

$$
\int_{\mathbb{U}^{N} \times \mathbb{R}^{N}} f=\int_{\mathbb{U}^{N} \times \mathbb{R}^{N}} g
$$

Define the $H$ functional (negative of the entropy) and the Kullback relative information by

$$
H(f)=\int_{\mathbb{T}^{N} \times \mathbb{R}^{N}} f \log f, \quad H(f \mid g)=\int_{\mathbb{T}^{N} \times \mathbb{R}^{N}} f \log \frac{f}{g} .
$$

Proposition 4. The well-known Csiszár-Kullback inequality asserts

$$
H(f g) \geq \frac{1}{4}\|f-g\|_{L^{1}(x, v)}^{2}
$$

if $f$ and $g$ are two distributions on $\mathbb{T}^{N} \times \mathbb{R}^{N}$. Moreover, if $f$ is the solution of (1), (2) and satisfies (7), then

$$
H(f \mid M)=H(f)-H(M)-\frac{1}{2} \int_{\mathbb{T}^{N}}|\nabla \phi|^{2} .
$$

Proof. Define $\varphi(h)=h \log (h)$; then since $\int f=\int g=1$, we have

$$
\begin{aligned}
H(f \mid g) & =\int f \log \frac{f}{g}=\int f \log f-f \log g-f+g \\
& =\int f \log f-g \log g-(\log g+1)(f-g) \\
& =\int \varphi(f)-\varphi(g)-\varphi^{\prime}(g)(f-g) \\
& =\frac{1}{2} \int \varphi^{\prime \prime}(h)|f-g|^{2} \\
& =\frac{1}{2} \int \frac{1}{h}|f-g|^{2},
\end{aligned}
$$

where $h$ stands for a positive function between $f$ and $g$. The last equality is obtained by using second-order Taylor expansion. By Hölder's inequality, we have

$$
\begin{aligned}
& \int|f-g| h^{-1 / 2} h^{1 / 2} \\
& \quad \leq\left(\int \frac{1}{h}|f-g|^{2}\right)^{1 / 2}\left(\int h\right)^{1 / 2}, \quad \forall h>0 .
\end{aligned}
$$

Since $h$ lies between $f$ and $g$, notice that distributions $f, g$ are nonnegative; thus $h \leq f+g$. We have

$$
\int \frac{1}{h}|f-g|^{2} \geq \frac{\left(\int|f-g|\right)^{2}}{\int(f+g)}=\frac{1}{2}\left(\int|f-g|\right)^{2},
$$

and (19) is obtained. Equation (20) follows directly from (7).

We now state the quantitative version of $H$-theorem. See [10] for the proof.

Theorem 5 (Quantitative $H$-Theorem). If $(f)_{t \geq 0}$ is a smooth solution of the VPB equation (1), (2), then the $H$ functional $H(f)$ is nonincreasing as a function of $t$, and the decreasing rate

$$
\frac{d}{d t} H(f)=-\int_{\mathbb{T}^{N}} D(f(x, \cdot)) d x
$$

where

$$
D(f)=\frac{1}{4} \int_{\mathbb{R}^{N} \times \mathbb{R}^{N} \times \mathbb{S}^{N-1}}\left(f^{\prime} f_{*}^{\prime}-f f_{*}\right) \log \frac{f^{\prime} f_{*}^{\prime}}{f f_{*}} B d \sigma d v d v_{*}
$$

is a positive definite functional.

Moreover, if the collision kernel $q$ satisfies (10), and $f$ complies with (12), then

$$
\begin{gathered}
D(f) \geq K_{\epsilon}\left(\int_{\mathbb{R}^{N}} f \log \frac{f}{M_{[\rho, u, T]}}\right)^{1+\epsilon}, \\
-\frac{d}{d t} H(f) \geq K_{H} H\left(f \mid M_{[\rho, u, T]}\right)^{1+\epsilon} .
\end{gathered}
$$

The only set that can make D vanish is the local Maxwellian state.

We state some notations here for the fluency of description. Let $A$ and $B$ be matrices; let the operation $A: B=$ $\sum_{i j} A_{i j} B_{i j}$. For a vector-valued function $u$, the divergence is

$$
\nabla_{x} \cdot u=\sum_{i} \frac{\partial u_{i}}{\partial x_{i}}
$$

the elements of gradient matrix $\nabla_{x} u$ satisfy

$$
\left(\nabla_{x} u\right)_{i j}=\frac{\partial u_{j}}{\partial x_{i}}
$$

the symmetric part of $\nabla u$ is

$$
\nabla_{x}^{\mathrm{sym}} u=\frac{\nabla_{x} u+\left(\nabla_{x} u\right)^{T}}{2}
$$

and the traceless part of $\nabla_{x}^{\text {sym }} u$ is symbolized by $\left\{\nabla_{x} u\right\}$ :

$$
\left\{\nabla_{x} u\right\}=\nabla_{x}^{\text {sym }} u-\frac{\nabla_{x} \cdot u}{N} I_{N} .
$$


We expect to estimate decay rate of the distance between $f$ and $M$, and the distance is measured by Kullback relative information. By using conservation laws, a direct computation will show that the relative information between $f$ and $M$ can be decomposed into a purely hydrodynamic part and a purely kinetic part:

$$
\begin{aligned}
H(f \mid M) & =\mathscr{H}(\rho, u, T)+H\left(f \mid M_{[\rho, u, T]}\right), \\
\mathscr{H}(\rho, u, T)= & \int_{T^{N}} \rho \log \rho+\frac{N}{2} \int_{T^{N}} \rho(T-\log T-1) \\
& +\int_{T^{N}} \rho \frac{|u|^{2}}{2} \\
= & : \mathscr{H}(\rho \mid 1)+\mathscr{H}(T \mid 1)+\mathscr{H}(u \mid 0),
\end{aligned}
$$

where

$$
\begin{gathered}
\mathscr{H}(u \mid 0)=\int \rho \frac{|u|^{2}}{2}, \\
\mathscr{H}(\rho \mid 1)=\int_{T^{N}} \rho \log \rho=\int_{T^{N}} \rho \log \rho-\rho+1
\end{gathered}
$$

are nonnegative since $\rho \log \rho-\rho+1$ is convex with the minimum zero at $\rho=1$.

Moreover, denote $\Psi(X)=(N / 2)(X-\ln X-1)$; we can further decompose $\mathscr{H}(T \mid 1)$ into

$$
\mathscr{H}(T \mid 1)=\mathscr{H}(T \mid\langle T\rangle)+\mathscr{H}(\langle T\rangle \mid 1),
$$

where

$$
\begin{gathered}
\mathscr{H}(T \mid\langle T\rangle)=\int \rho \Psi(T)-\Psi(\langle T\rangle), \\
\mathscr{\ell}(\langle T\rangle \mid 1)=\Psi(\langle T\rangle)
\end{gathered}
$$

It is easy to check that each of the previous terms is nonnegative by using Jensen's inequality and convexity of functions $\Psi(X)$.

It is easy to verify the following.

Lemma 6. Use the previously mentioned notations; then one has the following additivity roles:

$$
\begin{gathered}
H\left(f \mid M_{[\rho, u, T]}\right)+\mathscr{H}(T \mid\langle T\rangle)=H\left(f \mid M_{[\rho, u,\langle T\rangle]}\right), \\
H\left(f \mid M_{[\rho, u,\langle T\rangle]}\right)+\frac{1}{\langle T\rangle} \mathscr{H}(u \mid 0)=H\left(f \mid M_{[\rho, 0,\langle T\rangle]}\right), \\
H\left(f \mid M_{[\rho, 0,\langle T\rangle]}\right)+\mathscr{H}(\langle T\rangle \mid 1)+\left(1-\frac{1}{\langle T\rangle}\right) \mathscr{H}(u \mid 0) \\
=H\left(f \mid M_{[\rho, 0,1]}\right), \\
H\left(f \mid M_{[\rho, 0,1]}\right)+\mathscr{H}(\rho \mid 1)=H(f \mid M) .
\end{gathered}
$$

Moreover, one has

$$
\begin{gathered}
H\left(f \mid M_{[\rho, u, T]}\right) \geq K_{I}\left\|f-M_{[\rho, u, T]}\right\|_{L^{2}}^{2(1+\epsilon)}, \\
H\left(f \mid M_{[\rho, u,\langle T\rangle]}\right) \geq K_{I}\left\|f-M_{[\rho, u,\langle T\rangle]}\right\|_{L^{2}}^{2(1+\epsilon)}, \\
H\left(f \mid M_{[\rho, 0,\langle T\rangle]}\right) \geq K_{I}\left\|f-M_{[\rho, 0,\langle T\rangle]}\right\|_{L^{2}}^{2(1+\epsilon)}, \\
H\left(f \mid M_{[\rho, 0,1]}\right) \geq K_{I}\left\|f-M_{[\rho, 0,1]}\right\|_{L^{2}}^{2(1+\epsilon)} .
\end{gathered}
$$

Here nonnegative terms $\mathscr{H}(\rho \mid 1), \mathscr{H}(u \mid 0), \mathscr{H}(T \mid\langle T\rangle)$, $\mathscr{H}(\langle T\rangle \mid 1)$ are parts of the relative entropy, $K_{I}>0$.

Proof. Additivity rules can be verified by direct computation. By using Csiszár-Kullback inequality and the interpolation from $L^{2}$ into $L^{1}$, we can get (36). See [4] or [5] for more details.

Now we assert the key lemma of the paper, which asserts the instability of hydrodynamic descriptions for $f$.

Lemma 7. The following four second-order differential inequalities hold:

$$
\begin{aligned}
& \frac{d^{2}}{d t^{2}} \| f-M_{[\rho, u, T]} \|_{L^{2}(x, v)}^{2} \\
& \geq K_{1}\left[\int_{T^{N}}|\nabla T(x)|^{2} d x+\int_{T^{N}}|\{\nabla u(x)\}|^{2} d x\right] \\
&-\frac{C_{1}}{\delta_{1}^{1-\epsilon}}\left(\left\|f-M_{[\rho, u, T]}\right\|_{L^{2}}^{2}\right)^{1-\epsilon}-\delta_{1} H(f \mid M), \\
& \frac{d^{2}}{d t^{2}}\left\|f-M_{[\rho, u,\langle T\rangle]}\right\|_{L^{2}(x, v)}^{2} \\
& \geq K_{2} \int_{T^{N}}\left|\nabla^{s y m} u\right|^{2} d x \\
&-\frac{C_{2}}{\delta_{2}^{1-\epsilon}}\left(\left\|f-M_{[\rho, u,\langle T\rangle]}\right\|_{L^{2}}^{2}\right)^{1-\epsilon}-\delta_{2} H(f \mid M), \\
& \frac{d^{2}}{d t^{2}}\left\|f-M_{[\rho, 0,\langle T\rangle]}\right\|_{L^{2}(x, v)}^{2} \\
& \geq K_{3}\left[\int_{T^{N}}|\nabla \phi|^{2} d x+\int_{T^{N}}|\nabla \rho|^{2} d x\right] \\
&-\frac{C_{3}}{\delta_{3}^{1-\epsilon}}\left(\left\|f-M_{[\rho, 0,\langle T\rangle]}\right\|_{L^{2}}^{2}\right)^{1-\epsilon}-\delta_{3} H(f \mid M), \\
& \frac{d^{2}}{d t^{2}} \| f--M_{[\rho, 0,1]} \|_{L^{2}(x, v)}^{2} \\
& \geq K_{4}\left[\int_{T^{N}}|\nabla \phi|^{2} d x+\int_{T^{N}}|\nabla \rho|^{2} d x\right] \\
& \delta_{4}^{1-\epsilon}\left(\left\|f-M_{[\rho, 0,1]}\right\|_{L^{2}}^{2}\right)^{1-\epsilon}-\delta_{4} H(f \mid M) .
\end{aligned}
$$

Here $\delta_{1}, \delta_{2}, \delta_{3}, \delta_{4}$ are small enough constants, and all constants are positive. 
Roughly speaking, the previous inequalities show that $f$ cannot stay near local Maxwellian states. The gradient of $T$ prevents $f$ from staying close to $M_{[\rho, u, T]}$ for long; the symmetric gradient of $u$ prevents $f$ from staying close to $M_{[\rho, u,\langle T\rangle]}$ for long; finally, the gradient of $\rho$ prevents $f$ from staying close to $M_{[\rho, 0,\langle T\rangle]}$ and $M_{[\rho, 0,1]}$. It left $M=M_{[1,0,1]}$ as the only stable state.

To prove Lemma 7, the following lemma is needed, whose proof can be found in [4].

Lemma 8. Let $h$ be a smooth function of $x$, $v$. Then, for all multi-indexes $\alpha, \beta$, and for all $\eta<1$,

$$
\int\left(v^{\alpha} \partial_{x, v}^{\beta} h\right)^{2} d v d x \leq\|h\|_{H_{|\alpha| / \eta}^{|\beta|}}^{2 \eta}\|h\|_{H^{|\beta| / \eta}}^{2 \eta(1-\eta)}\|h\|_{L^{2}}^{2(1-\eta)^{2}} .
$$

Proof of Lemma 7. Most of the proof is similar to that in $[4,5]$; the only difference is in estimating terms with $\phi$. We will only prove (39) as an example of how to estimate terms with $\phi$. We have

$$
\begin{aligned}
\frac{d^{2}}{d t^{2}}\left\|f-M_{[\rho, 0,\langle T\rangle]}\right\|_{L^{2}}^{2} & \\
= & 2 \int\left(\frac{\partial}{\partial t}\left(f-M_{[\rho, 0,\langle T\rangle]}\right)\right)^{2} d v d x \\
& +2 \int\left(f-M_{[\rho, 0,\langle T\rangle]}\right) \frac{\partial^{2}}{\partial t^{2}}(f-g) d v d x \\
= & A+B .
\end{aligned}
$$

At the moment when $f=M_{[\rho, 0,\langle T\rangle]}, B$ vanishes, so we only need to estimate $A$ :

$$
\begin{aligned}
\frac{\partial}{\partial t}\left(f-M_{[\rho, 0,\langle T\rangle]}\right)= & -v \cdot \nabla_{x} f-\nabla_{x} \phi \cdot \nabla_{v} f+Q(f, f) \\
& -\frac{\partial}{\partial t} M_{[\rho, 0,\langle T\rangle]} \\
= & -\left(\frac{\partial}{\partial t}+v \cdot \nabla_{x}+\nabla_{x} \phi \cdot \nabla_{v}\right) M_{[\rho, 0,\langle T\rangle]} .
\end{aligned}
$$

From (1) we have

$$
\begin{gathered}
\rho_{t}+\nabla_{x} \cdot(\rho u)=0, \\
(\rho u)_{t}+\nabla_{x} \cdot\left(\rho u \otimes u+\rho T I_{N}+D\right)-\rho \nabla_{x} \phi=0, \\
\left(\rho|u|^{2}+N \rho T\right)_{t}+\nabla_{x} \\
\cdot\left(\rho|u|^{2} u+(N+2) \rho u T+2 D u+2 R\right) \\
-2 \rho u \cdot \nabla_{x} \phi=0 .
\end{gathered}
$$

Here, $D$ and $R$ are matrix-valued and vector-valued functions, respectively, defined by

$$
\begin{array}{rl}
D_{i j}(x)=\int_{R^{N}} & f(x, v) \\
& \times\left[(v-u)_{i}(v-u)_{j}-\frac{|v-u|^{2}}{N} \delta_{i j}\right] d v, \\
R(x)= & \int_{R^{N}} f(x, v) \frac{|v-u|^{2}}{2}(v-u) d v .
\end{array}
$$

Then, we obtain

$$
\begin{gathered}
\left(\partial_{t}+u \cdot \nabla\right) \rho+\rho \nabla \cdot u=0, \\
\left(\partial_{t}+u \cdot \nabla\right) u+\nabla T+\frac{T \nabla \rho}{\rho}+\frac{\nabla \cdot D}{\rho}-\nabla_{x} \phi=0, \\
\left(\partial_{t}+u \cdot \nabla\right) T+\frac{2 T}{N} \nabla \cdot u+\frac{2}{\rho N}(\nabla u: D+\nabla \cdot R)=0 .
\end{gathered}
$$

Also, we get

$$
\begin{aligned}
\partial_{t}\langle T\rangle= & {\left[\int\left(\partial_{t} \rho\right) T+\int \rho\left(\partial_{t} T\right)\right] } \\
= & {\left[-\int \nabla \cdot(\rho u) T-\int \rho u \cdot \nabla T-\frac{2}{N} \int \rho T \nabla \cdot u\right.} \\
& \left.\quad-\frac{2}{N} \int \nabla u: D-\frac{2}{N} \int \nabla \cdot R\right] \\
= & {\left[-\frac{2}{N} \int \rho T \nabla \cdot u-\frac{2}{N} \int \nabla u: D\right] . }
\end{aligned}
$$

Then the equations of $M_{[\rho, 0,\langle T\rangle]}$ can be stated as follows:

$$
\begin{aligned}
& \left(\partial_{t}+v \cdot \nabla_{x}+\nabla_{x} \phi \cdot \nabla_{v}\right) M_{[\rho, 0,\langle T\rangle]} \\
& =M_{[\rho, 0,\langle T\rangle]} \\
& \quad \times\left\{\left[\frac{\partial_{t} \rho}{\rho}-\frac{N}{2} \frac{\partial_{t}\langle T\rangle}{\langle T\rangle}\right]\right. \\
& \left.\quad+v \cdot\left[\frac{\nabla \rho}{\rho}-\frac{\nabla_{x} \phi}{\langle T\rangle}\right]+|v|^{2}\left[\frac{\partial_{t}\langle T\rangle}{2\langle T\rangle^{2}}\right]\right\} .
\end{aligned}
$$

From (46) and (47), we have

$$
\begin{aligned}
\partial_{t}\left(f-M_{[\rho, 0,\langle T\rangle]}\right) \\
=-M_{[\rho, 0,\langle T\rangle]} \\
\quad \times\left\{\left[\frac{\partial_{t} \rho}{\rho}-\frac{N}{2} \frac{\partial_{t}\langle T\rangle}{\langle T\rangle}\right]\right. \\
\left.+v \cdot\left[\frac{\nabla \rho}{\rho}-\frac{\nabla_{x} \phi}{\langle T\rangle}\right]+|v|^{2}\left[\frac{\partial_{t}\langle T\rangle}{2\langle T\rangle^{2}}\right]\right\} .
\end{aligned}
$$


Note that $M_{[\rho, 0,\langle T\rangle]},|v|^{2} M_{[\rho, 0,\langle T\rangle]},|v|^{2} M_{[\rho, 0,\langle T\rangle]}$ are linearly independent in weighted $L^{2}\left(\left(1 / M_{[\rho, 0,\langle T\rangle]}\right) d v\right)$ space. Therefore,

$$
\begin{aligned}
& \left.\frac{d^{2}}{d t^{2}}\left\|f-M_{[\rho, 0,\langle T\rangle]}\right\|_{L^{2}}^{2}\right|_{f=M_{[\rho, 0,\langle T\rangle]}} \\
& \geq \kappa \int_{T^{N}}\left|\frac{\nabla \rho}{\rho}-\frac{\nabla_{x} \phi}{\langle T\rangle}\right|^{2} d x \\
& =\kappa\left[\int\left|\frac{\nabla \rho}{\rho}\right|^{2}+\int\left|\frac{\nabla_{x} \phi}{\langle T\rangle}\right|^{2}-2 \int \frac{\nabla \rho \cdot \nabla \phi}{\rho\langle T\rangle}\right],
\end{aligned}
$$

where

$$
\begin{aligned}
-2 \int \frac{\nabla \rho \cdot \nabla \phi}{\rho\langle T\rangle} & =-\frac{2}{\langle T\rangle} \int \nabla \ln \rho \cdot \nabla \phi \\
& =\frac{2}{\langle T\rangle} \int \ln \rho \Delta \phi=\frac{2}{\langle T\rangle} \int \ln \rho(\rho-1) .
\end{aligned}
$$

It is easy to verify the convexity and nonnegativity of $\ln \rho(\rho-$ 1). Therefore,

$$
\begin{aligned}
& \left.\frac{d^{2}}{d t^{2}}\left\|f-M_{[\rho, 0,\langle T\rangle]}\right\|_{L^{2}}^{2}\right|_{f=M_{[\rho, 0,\langle T\rangle]}} \\
& \quad \geq \kappa\left[\int\left|\frac{\nabla \rho}{\rho}\right|^{2}+\int\left|\frac{\nabla_{x} \phi}{\langle T\rangle}\right|^{2}\right] \\
& \geq K_{3}\left[\int_{T^{N}}|\nabla \rho|^{2} d x+\int_{T^{N}}\left|\nabla_{x} \phi\right|^{2} d x\right] .
\end{aligned}
$$

When $f$ does not coincide with $M_{[\rho, 0,\langle T\rangle]}$, we need to estimate two terms $A$ and $B$ of (42) separately. The detailed calculation can be found in $[4,5]$. Also, we just emphasize the estimates for terms with $\phi$ here.

Notice that, when estimating $B$, we need to control $\left\|\partial^{2} f / \partial t^{2}\right\|_{L^{2}}$ by $\|f-M\|_{L^{2}}^{1-\alpha}$. Substitute the Vlasov-PoissonBoltzmann equation (1) into $\partial^{2} f / \partial t^{2}$; we get terms of $\phi$.

(a) $L^{2}$ norm estimate of $\nabla_{x} \phi_{t} \cdot \nabla_{v} f$.

It is obvious that

$$
\begin{aligned}
& \left\|\nabla_{x} \phi_{t} \cdot \nabla_{v} f\right\|_{L^{2}} \\
& \leq\left\|\nabla_{x} \phi_{t} \cdot \nabla_{v}(f-M)\right\|_{L^{2}}+\left\|\nabla_{x} \phi_{t} \cdot \nabla_{v} M\right\|_{L^{2}} \\
& \leq C\left(\int\left|\nabla_{v}(f-M)\right|^{2} d x d v\right)^{1 / 2} \\
& \quad+C\left(\int\left|\nabla_{x} \phi_{t}\right|^{2} d x\right)^{1 / 2} .
\end{aligned}
$$

The first term is bounded by $C\|f-M\|_{L^{2}}^{1-\alpha}$ by interpolation lemma. As for the second term, since

$$
\begin{aligned}
\int\left|\nabla_{x} \phi_{t}\right|^{2} d x & =-\int \Delta \phi_{t} \cdot \phi_{t} d x=-\int \rho_{t} \phi_{t} d x \\
& =\int \nabla_{x} \cdot(\rho u) \phi_{t} d x=-\int \rho u \cdot\left(\nabla_{x} \phi_{t}\right) d x \\
& \leq\left(\int \rho^{2} u^{2} d x\right)^{1 / 2}\left(\int\left|\nabla_{x} \phi_{t}\right|^{2} d x\right)^{1 / 2}
\end{aligned}
$$

we have

$$
\begin{aligned}
\int\left|\nabla_{x} \phi_{t}\right|^{2} d x & \leq C \int \rho^{2} u^{2} d x \leq C \mathscr{H}(u \mid 0) \\
& \leq C H(f \mid M) \leq C\|f-M\|_{L^{2}}^{2} .
\end{aligned}
$$

Hence, $\left\|\nabla_{x} \phi_{t} \cdot \nabla_{v} f\right\|_{L^{2}} \leq C\|f-M\|_{L^{2}}^{1-\alpha}$.

(b) $L^{2}$ norm estimate of $\left(v \otimes \nabla_{v} M\right):\left(\nabla_{x}^{2} \phi\right)$.

Note that $M$ is a Gaussian distribution, so that $M^{2}$ times any polynomials of $v$ is integrable:

$$
\begin{aligned}
\|(v & \left.\otimes \nabla_{v} M\right):\left(\nabla_{x}^{2} \phi\right) \|_{L^{2}}^{2} \\
& =\left\|(v \otimes v):\left(\nabla_{x}^{2} \phi\right) M\right\|_{L^{2}}^{2} \\
& =\sum_{i, j, k, l} \int v_{i} v_{j} v_{k} v_{l} M^{2} \partial_{x_{i} x_{j}} \phi \partial_{x_{k} x_{l}} \phi d v d x \\
& \leq C \sum_{i, j} \int \partial_{x_{i} x_{i}} \phi \partial_{x_{j} x_{j}} \phi d x \\
& =C \int(\Delta \phi)^{2} d x=C \int|\rho-1|^{2} d x \\
& \leq C \mathscr{H}(\rho \mid 1) \leq C H(f \mid M) \leq C\|f-M\|_{L^{2}}^{2} .
\end{aligned}
$$

(c) $L^{2}$ norm estimate of $\left(\nabla_{x} \phi \otimes \nabla_{x} \phi\right): \nabla_{v}^{2} M$.

Similarly as in the previous argument, $M^{2}$ times any polynomials of $v$ is integrable. Also, $\phi$ and $\partial \phi$ are bounded by Schauder estimate because it is constrained by a Poisson equation.

Note that $\Delta \phi=\rho-1$; we have

$$
\begin{aligned}
\int_{T^{N}}|\nabla \phi|^{2} d x & =-\int \Delta \phi(\phi-\bar{\phi}) d x \\
& =-\int(\rho-1)(\phi-\bar{\phi}) d x \\
& \leq\left(\int(\rho-1)^{2}\right)^{1 / 2}\left(\int(\phi-\bar{\phi})^{2}\right)^{1 / 2} \\
& \leq K_{P}^{1 / 2}\left(\int(\rho-1)^{2}\right)^{1 / 2}\left(\int|\nabla \phi|^{2}\right)^{1 / 2} .
\end{aligned}
$$

Here, $K_{P}$ is the constant appearing in the Poincaré inequality, which is only relevant to the domain $T^{N}$. Thus,

$$
\int|\nabla \phi|^{2} \leq K_{P} \int(\rho-1)^{2} \leq K_{P}^{2} \int|\nabla \rho|^{2}
$$


Therefore,

$$
\begin{aligned}
& \left\|\left(\nabla_{x} \phi \otimes \nabla_{x} \phi\right): \nabla_{v}^{2} M\right\|_{L^{2}}^{2} \\
& =\left\|\left(\nabla_{x} \phi \otimes \nabla_{x} \phi\right):(v \otimes v-I) M\right\|_{L^{2}}^{2} \\
& =\sum_{i, j, k, l} \int \partial_{i} \phi \partial_{j} \phi \partial_{k} \phi \partial_{l} \phi\left(v_{i} v_{j}-\delta_{i j}\right) \\
& \quad \times\left(v_{k} v_{l}-\delta_{k l}\right) M^{2} d v d x \\
& \leq C \sum_{i, j} \int\left(\partial_{i} \phi \partial_{j} \phi\right)^{2} d x \leq C \sum_{i} \int\left(\partial_{i} \phi\right)^{2} d x \\
& =C \int|\nabla \phi|^{2} d x \leq C \int|\rho-1|^{2} d x \leq C \mathscr{H}(\rho \mid 1) \\
& \leq C H(f \mid M) \leq C\|f-M\|_{L^{2}}^{2} .
\end{aligned}
$$

(d) $L^{2}$ norm estimate of $Q^{\text {sym }}\left(f, \nabla_{x} \phi \cdot \nabla_{v} M\right)$.

From the momentum and energy conservation of particle collisions, it is easy to verify that

$$
Q^{\text {sym }}\left(M, v_{i} M\right)=0 .
$$

Thus,

$$
Q^{\text {sym }}\left(f, \nabla_{x} \phi \cdot \nabla_{v} M\right)=Q^{\text {sym }}\left(f-M, \nabla_{x} \phi \cdot v M\right) .
$$

Then, using our continuity assumption (11) on $Q(g, h)$ and the interpolation Lemma 8 , we can estimate $L^{2}$ norm of $Q^{\text {sym }}\left(f, \nabla_{x} \phi \cdot \nabla_{v} M\right)$ by $\|f-M\|_{L^{2}}^{1-\alpha}$. Therefore, we have

$$
\begin{aligned}
& \forall 0<\alpha<\eta<1, \\
& \left\|\frac{\partial^{2} f}{\partial t^{2}}\right\|_{L^{2}} \leq C_{\alpha}\|f-M\|_{L^{2}}^{1-\alpha} \leq C_{\eta}\|f-M\|_{L^{1}}^{1-\eta} .
\end{aligned}
$$

The rest of the proof is similar to that in [5]. Now we complete the proof of the lemma.

Notice that there is the symmetric gradient of $u$ in (38); the next lemma can provide a method to control this term.

Lemma 9. One has the Korn-type inequality:

$$
\int_{T^{N}}\left|\nabla^{s y m} u\right|^{2} d x \geq K_{K} \int_{\Omega}|\nabla u|^{2} d x
$$

and the following Poincaré-type inequalities:

$$
\begin{gathered}
\int_{T^{N}}|\nabla T|^{2} d x \geq K_{T} \mathscr{H}(T \mid\langle T\rangle), \\
\int_{T^{N}}|\nabla u|^{2} d x \geq K_{u} \mathscr{H}(u \mid 0), \\
\int_{T^{N}}|\nabla \rho|^{2} d x \geq K_{\rho} \mathscr{H}(\rho \mid 1) .
\end{gathered}
$$

Here all constants are positive.
Lemma 10. One has estimates on damping of hydrodynamic oscillations with $C_{S}>0$,

$$
\begin{aligned}
& \mid \frac{d}{d t} \mathscr{H}(\rho \mid 1), \frac{d}{d t} \mathscr{H}(u \mid 0), \frac{d}{d t} \mathscr{H}(\langle T\rangle \mid 1), \\
& \quad \frac{d}{d t} \mathscr{H}(T \mid\langle T\rangle) \mid \\
& \leq C_{S} H(f \mid M)^{1-\epsilon} .
\end{aligned}
$$

See [4] or [5] for the proof of the previous two lemmas. Inequalities of Lemma 9 provide estimates of the right-hand side of second-order differential inequalities in Lemma 7. Lemma 10 provides the decay rate for hydrodynamic oscillations.

\section{Proof of the Main Result}

Use the previous lemmas; we are now ready to prove Theorem 2. The main idea is similar to that in [5]; for convenience of the reader, we restate the sketch of the proof and make it more complete by proving Lemma 11.

From $H$-theorem (Theorem 5), the convergence rate of $H(f)$ to $H(M)$ is determined by entropy production functional $D(f)$. But there are many local Maxwellians, which make our entropy production functional $D(f)$ vanish. Therefore it is impossible to get a uniform lower bound on the entropy production. To overcome this difficulty, it is natural to estimate the average value of entropy production. Suppose that

$$
\alpha_{0}=H(f)-\left.H(M)\right|_{t=t_{0}} .
$$

We wish to find an upper bound on a duration $T_{0}$ (it is possible since $H(f)$ is monotone nonincreasing), such that

$$
H(f)-\left.H(M)\right|_{t=t_{0}+T_{0}}=\sigma \alpha_{0},
$$

where $\sigma \in(0,1)$ is fixed; say $\sigma=4 / 5$. Therefore, we have

$$
\frac{4}{5} \alpha_{0} \leq H(f)-H(M) \leq \alpha_{0}
$$

Lemma 11. Choose that $\epsilon>0$ is small enough, like $\epsilon<0.01$, if one can show

$$
T_{0} \leq C_{0}(\epsilon) \alpha_{0}^{-699 \epsilon}
$$

where $C_{0}$ depends on $\epsilon$ and the various constants appearing in lemmas of Section 2. Then

$$
H(f)-H(M)=O\left(t^{-1 / 700 \epsilon}\right)
$$

Proof. Fix $\epsilon>0$ sufficiently small. Denote $H(f)-H(M)$ by $g(t)$. It is not hard to prove the continuity of $g(t)$. From the boundedness of initial data $f_{0}$, we can denote $t_{0}:=0$, $\left.g(t)\right|_{t=0}=g(0)=: \beta_{0}$. It is sufficient to prove that, for all $t>0$, $t^{1 / 700 \epsilon} g(t)$ or equivalently $\operatorname{tg}(t)^{700 \epsilon}$ is uniformly bounded.

Define a sequence $\left\{t_{i}\right\}$, such that

$$
\left.g(t)\right|_{t=t_{i}}=\sigma^{i} \beta_{0} .
$$


Correspondingly, we can define $\left\{T_{i}\right\}, T_{i}=t_{i}-t_{i-1}$. From the estimate of $T_{0}$ in (69), we have

$$
T_{i} \leq C_{0}(\epsilon)\left(\sigma^{i-1} \beta_{0}\right)^{-699 \epsilon} .
$$

Therefore,

$$
t_{i}=\sum_{k=1}^{i} T_{k} \leq C_{0}(\epsilon)\left(\beta_{0}\right)^{-699 \epsilon} \frac{\sigma^{-699 \epsilon(i-1)}-1}{\sigma^{-699 \epsilon}-1} .
$$

It is obvious that $t_{i} \rightarrow \infty$, as $i \rightarrow \infty$.

For any $t>0$, we can find an interval such that $t \in$ $\left[t_{i-1}, t_{i}\right]$. Now we are ready to estimate $\operatorname{tg}(t)^{700 \epsilon}$. From the monotonicity of $g(t)$, we have

$$
\begin{aligned}
\operatorname{tg}(t)^{700 \epsilon} \leq & t_{i} g\left(t_{i-1}\right)^{700 \epsilon} \\
\leq & C_{0}(\epsilon)\left(\beta_{0}\right)^{-699 \epsilon} \frac{\sigma^{-699 \epsilon(i-1)}-1}{\sigma^{-699 \epsilon}-1} \\
& \times\left(\sigma^{i-1} \beta_{0}\right)^{700 \epsilon} \leq C,
\end{aligned}
$$

where the constant is independent of $i$, since $\sigma<1$ is fixed and $\epsilon>0$ can be chosen to be sufficiently small.

Once condition (69) is proved, the main theorem is a direct consequence of $H(f \mid M)=O(H(f)-H(M))$. Indeed, from (58) and (20), we have

$$
\begin{aligned}
\int|\nabla \phi|^{2} \leq K_{P} \int(\rho-1)^{2} \leq K^{\prime} \mathscr{H}(\rho \mid 1) \leq K^{\prime \prime} H(f \mid M), \\
H(f \mid M) \leq H(f)-H(M)=H(f \mid M)+\int \frac{1}{2}|\nabla \phi|^{2} \\
\leq\left(1+\frac{K^{\prime \prime}}{2}\right) H(f \mid M) .
\end{aligned}
$$

Therefore, it remains to prove condition (69). Detailed proof can be found in the last part of [5] for Vlasov-PoissonBoltzmann equations; we only describe the idea of the proof for the completion of this paper. Consider

$$
\frac{4}{5} \alpha_{0} \leq H(f)-H(M) \leq \alpha_{0},
$$

on interval $I:=\left[t_{0}, t_{0}+T_{0}\right]$; that is, $H(f)-H(M)$ has variation $\alpha_{0} / 5$. In order to prove (69), it is sufficient to prove that the average value of $-(d / d t) H(f)$ on interval $I$ satisfies

$$
\langle-\dot{H}(f)\rangle_{I} \geq \frac{C \alpha_{0}}{C_{0}(\epsilon) \alpha_{0}^{-699 \epsilon}}=C \alpha_{0}^{1+699 \epsilon} .
$$

Now we proceed the proof of Theorem 2 step by step.

(1) $I_{G}$ : Subinterval of $I$ Where $H\left(f \mid M_{[\rho, u, T]}\right)$ Is Large. From quantitative $H$-Theorem $5,\langle-\dot{H}(f)\rangle_{I_{G}}$ can be estimated directly on subinterval of $I$ where $H\left(f \mid{ }_{[\rho, u, T]}\right)$ is large. The subinterval can be called $I_{G}$, which means good interval. Other interval is called $I_{B}$, bad interval.
Notice the entropy additivity rules in Lemma 6; we actually have

$$
\begin{aligned}
H(f)-H(M)= & H(f \mid M)+\int \frac{1}{2}|\nabla \phi|^{2} \\
\leq & \left(1+\frac{K^{\prime}}{2}\right) \mathscr{H}(\rho \mid 1)+\mathscr{H}(u \mid 0) \\
& +\mathscr{H}(T \mid\langle T\rangle) \\
& +\mathscr{H}(\langle T\rangle \mid 1)+H\left(f \mid M_{[\rho, u, T]}\right) .
\end{aligned}
$$

(2) $I_{B G}$ : Subinterval of $I_{B}$ Where $\mathscr{H}(T \mid\langle T\rangle)$ Is Large. On interval $I_{B}, H\left(f \mid M_{[\rho, u, T]}\right)$ is small, while $H(f)-H(M)$ has lower bound $(4 / 5) \alpha_{0}$. Then by entropy additivity rules, we must have that $\mathscr{H}(\rho \mid 1)+\mathscr{H}(u \mid 0)+\mathscr{H}(T \mid\langle T\rangle)+\mathscr{H}(\langle T\rangle \mid 1)$ cannot be small.

Denote the subinterval of $I_{B}$ by $I_{B G}$ where $\mathscr{H}(T$ । $\langle T\rangle)$ is large. Then from the Poincaré-type inequalities of Lemma 9, we have that $\int_{T^{N}}|\nabla T|^{2} d x$ is large. Therefore, the right hand side of (37) is large. By an argument for secondorder differential inequalities (Lemma 12 of Desvillettes and Villani in [4]), we can conclude that either the average value of $\left\|f-M_{[\rho, u, T]}\right\|_{L^{2}}$ is large (so is $H\left(f \mid M_{[\rho, u, T]}\right)$ ) or the length of interval is small enough to be absorbed. $H$-theorem then asserts that average value $\langle-\dot{H}(f)\rangle_{I_{B G}}$ is large.

(3) $I_{B B G}$ : Subinterval of $I_{B B}$ Where $\mathscr{H}(u \mid 0)$ Is Large. On interval $I_{B B}, H\left(f \mid M_{[\rho, u, T]}\right), \mathscr{H}(T \mid\langle T\rangle)$ is small, while $H(f)-H(M)$ has lower bound $(4 / 5) \alpha_{0}$. Then similarly, $\mathscr{H}(\rho \mid$ $1)+\mathscr{H}(u \mid 0)+\mathscr{H}(\langle T\rangle \mid 1)$ cannot be small.

Denote the subinterval of $I_{B B}$ by $I_{B B G}$ where $\mathscr{H}(u$ । $0)$ is large. Then from the Poincaré-type and Korn-type inequalities of Lemma 9, we have that $\int\left|\nabla^{\mathrm{sym}} u\right|^{2} d x$ is large. Therefore, the right-hand side of (38) is large. By an argument for second-order differential inequalities (Lemma 12 of Desvillettes and Villani in [4]), we can conclude that either the average value of $\left\|f-M_{[\rho, u,\langle T\rangle]}\right\|_{L^{2}}$ is large (so is $H(f \mid$ $\left.M_{[\rho, u,\langle T\rangle]}\right)$ ) or the length of interval is small enough to be absorbed. But the first line of (35) shows that $H\left(f \mid M_{[\rho, u, T]}\right)$ must be large in average. $H$-theorem then asserts that average value $\langle-\dot{H}(f)\rangle_{I_{B B G}}$ is large.

(4) $I_{B B B G}$ : subinterval of $I_{B B B}$ where $\mathscr{H}(\langle T\rangle \mid 1)$ is large. On interval $I_{B B B}, H\left(f \mid M_{[\rho, u, T]}\right), \mathscr{H}(T \mid\langle T\rangle), \mathscr{H}(u \mid 0)$ is small, while $H(f)-H(M)$ has lower bound (4/5) $\alpha_{0}$. Then similarly, $\mathscr{H}(\rho \mid 1)+\mathscr{H}(\langle T\rangle \mid 1)$ cannot be small.

Denote the subinterval of $I_{B B B}$ by $I_{B B B G}$ where $\mathscr{H}(\langle T\rangle \mid 1)$ is large. From the conservation of energy, we have

$$
|\langle T\rangle-1|=\frac{2}{N} \mathscr{H}(u \mid 0)+\frac{1}{N} \int_{T^{N}}|\nabla \phi|^{2} d x .
$$

Because of the Lipschitz continuity of $\mathscr{H}(\langle T\rangle \mid 1)=\Psi(\langle T\rangle)$,

$$
\mathscr{H}(\langle T\rangle \mid 1) \leq L|\langle T\rangle-1| .
$$


Since $\mathscr{H}(u \mid 0)$ is sufficiently small in $I_{B B B G}$, therefore, (79) turns to be

$$
\mathscr{H}(\langle T\rangle \mid 1) \leq L|\langle T\rangle-1| \leq C \int_{T^{N}}|\nabla \phi|^{2} d x \leq C \mathscr{H}(\rho \mid 1) .
$$

Therefore, the right-hand side of (40) and (39) is large. By a similar argument as in previous subintervals, we can also show that average value $\langle-\dot{H}(f)\rangle_{I_{B B B G}}$ is large. By a careful calculation to absorb all the bad intervals into good ones, we can prove that average value $\langle-\dot{H}(f)\rangle_{I}$ is large on interval $I$. Thus, the whole proof is complete.

To conclude the paper, we remove the condition in Theorem 1 by making a crucial estimates on terms with $\phi$. The main differences with previous works [5] are in proving Lemma 7. We also complete the gap in the last part of $[4,5]$ by proving Lemma 11.

\section{Acknowledgment}

Project 11001066 is supported by the Natural Science Foundation of China.

\section{References}

[1] S. Ukai, "On the existence of global solutions of mixed problem for non-linear Boltzmann equation," Proceedings of the Japan Academy, vol. 50, pp. 179-184, 1974.

[2] R. E. Caflisch, "The Boltzmann equation with a soft potential. I. Linear, spatially-homogeneous," Communications in Mathematical Physics, vol. 74, no. 1, pp. 71-95, 1980.

[3] R. M. Strain and Y. Guo, "Exponential decay for soft potentials near Maxwellian," Archive for Rational Mechanics and Analysis, vol. 187, no. 2, pp. 287-339, 2008.

[4] L. Desvillettes and C. Villani, "On the trend to global equilibrium for spatially inhomogeneous kinetic systems: the Boltzmann equation," Inventiones Mathematicae, vol. 159, no. 2, pp. 245-316, 2005.

[5] L. Li, "On the trend to equilibrium for the Vlasov-PoissonBoltzmann equation," Journal of Differential Equations, vol. 244, no. 6, pp. 1467-1501, 2008.

[6] Y. Guo, "The Vlasov-Poisson-Boltzmann system near Maxwellians," Communications on Pure and Applied Mathematics, vol. 55, no. 9, pp. 1104-1135, 2002.

[7] R. M. Strain, "The Vlasov-Maxwell-Boltzmann system in the whole space," Communications in Mathematical Physics, vol. 268, no. 2, pp. 543-567, 2006.

[8] T. Yang, H. J. Yu, and H. J. Zhao, "Cauchy problem for the Vlasov-Poisson-Boltzmann system," Archive for Rational Mechanics and Analysis, vol. 182, no. 3, pp. 415-470, 2006.

[9] T. Yang and H. J. Zhao, "Global existence of classical solutions to the Vlasov-Poisson-Boltzmann system," Communications in Mathematical Physics, vol. 268, no. 3, pp. 569-605, 2006.

[10] C. Villani, "Cercignani's conjecture is sometimes true and always almost true," Communications in Mathematical Physics, vol. 234, no. 3, pp. 455-490, 2003. 


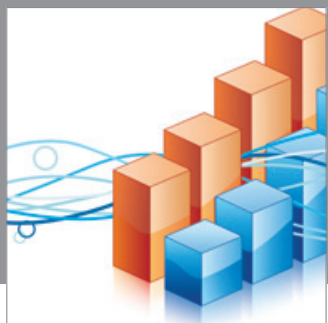

Advances in

Operations Research

mansans

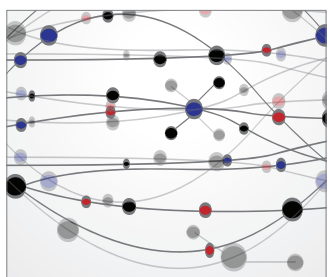

The Scientific World Journal
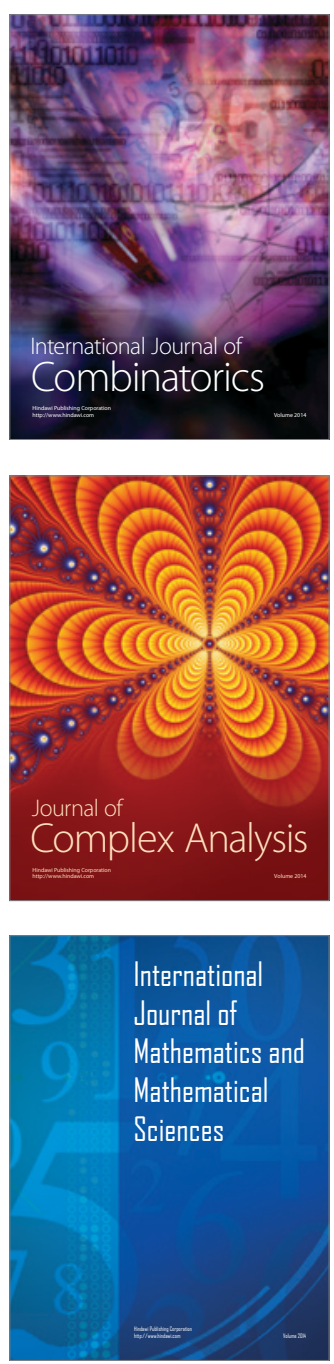
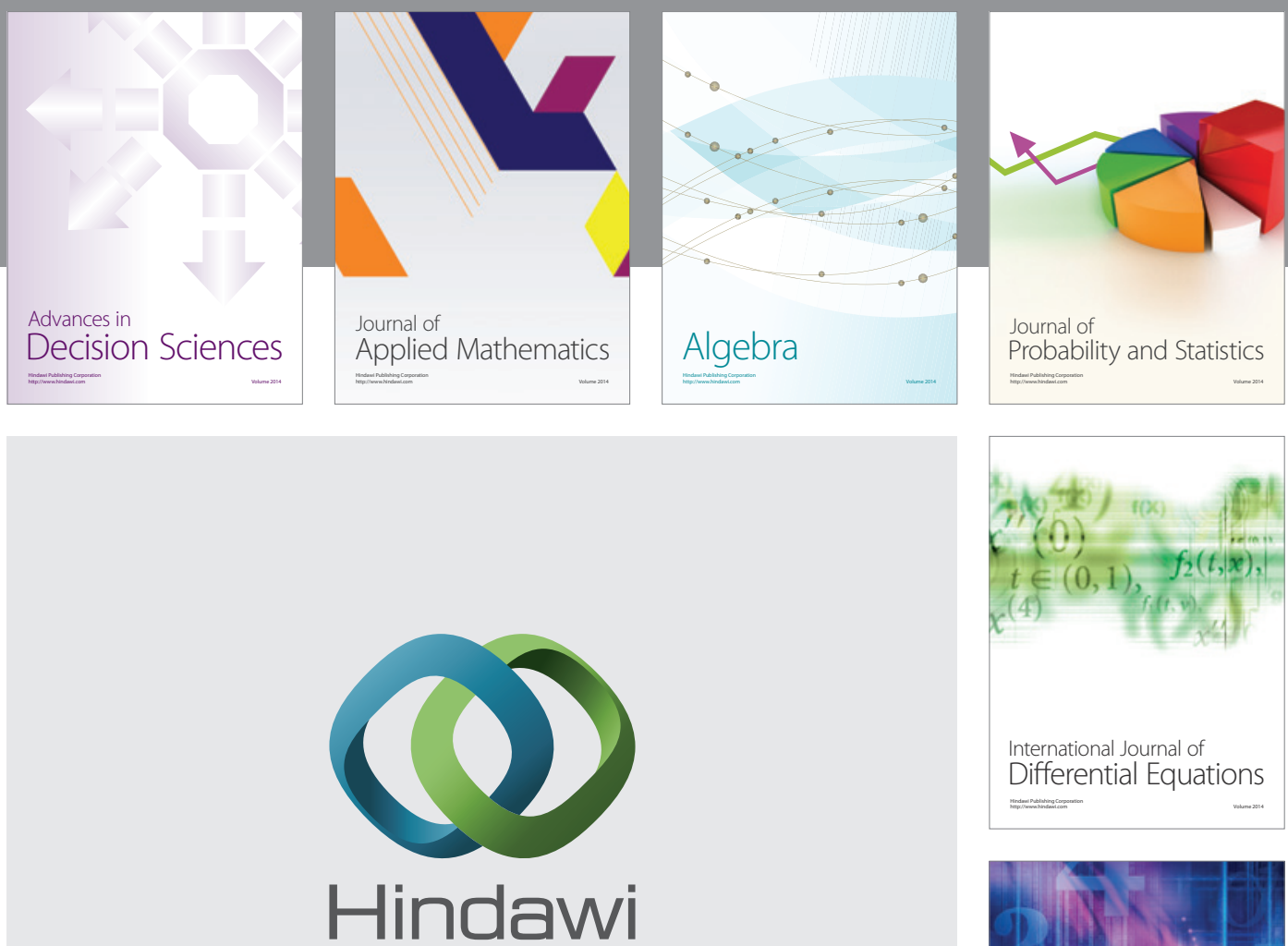

Submit your manuscripts at http://www.hindawi.com
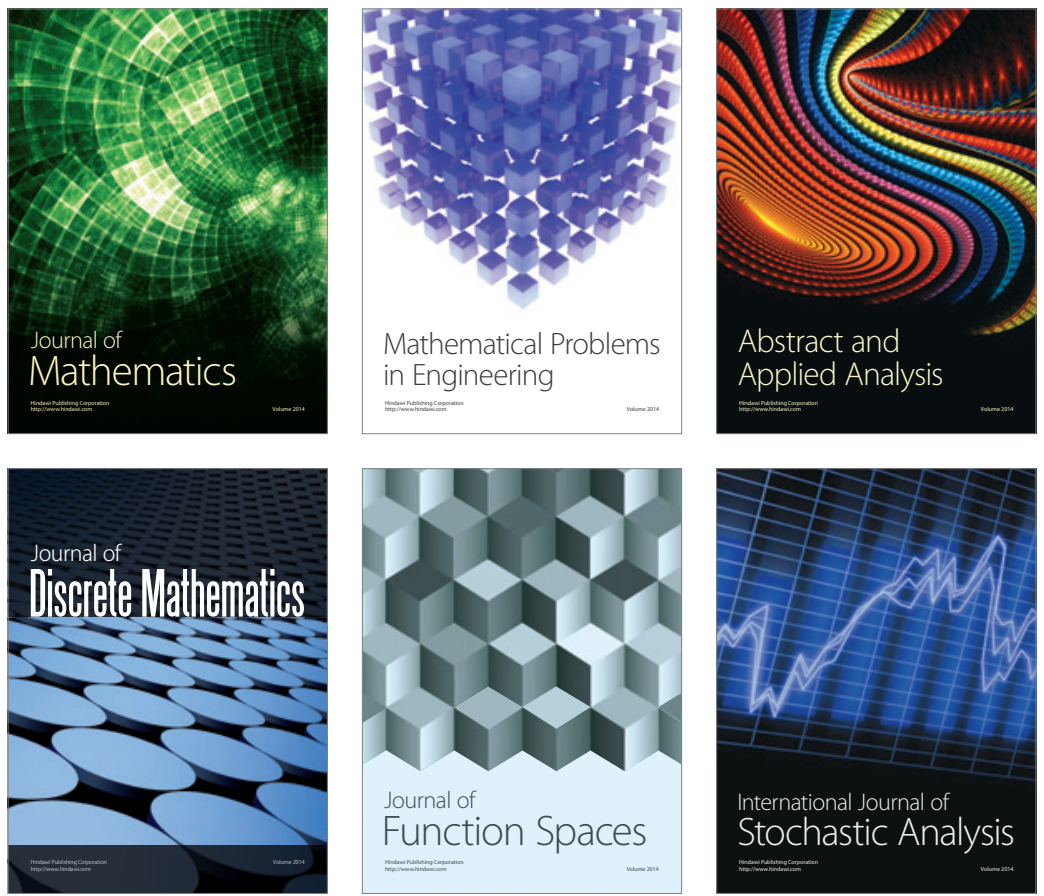

Journal of

Function Spaces

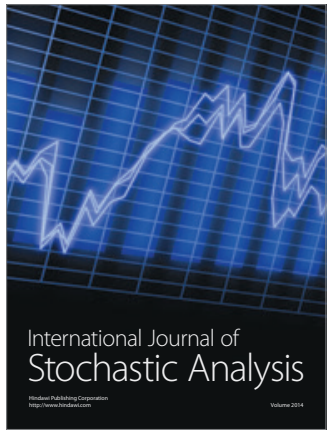

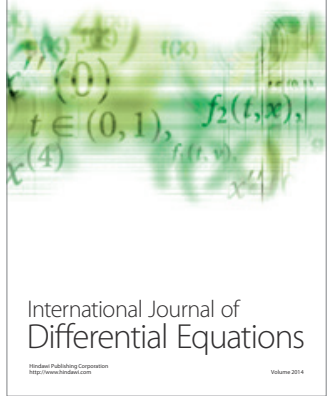
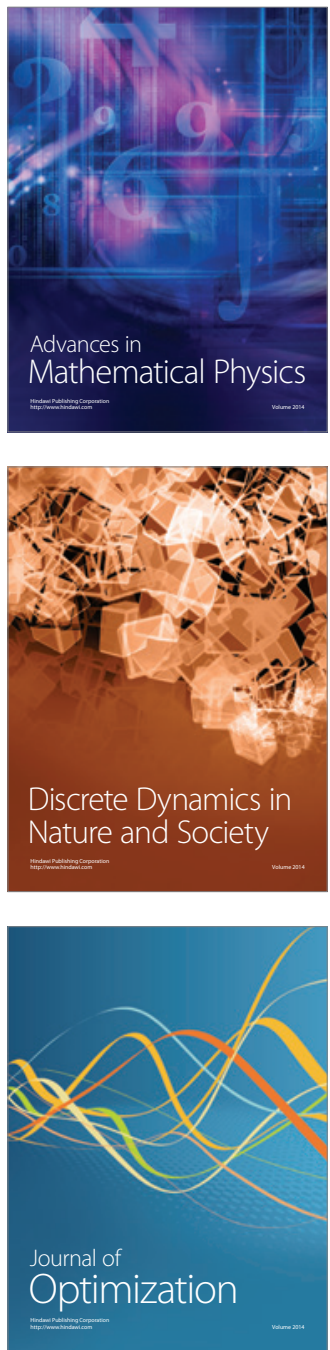